T. H. Steele, Department of Mathematics, Weber State University, Ogden, UT, 84408-1702. e-mail: thsteele@weber.edu

\title{
THE ESSENTIAL POINT SET OF A CONTINUOUS FUNCTION
}

\begin{abstract}
For continuous self maps of $[0,1]$, we extend M. K. Fort, Jr.'s notion of an essential fixed point to points generating nonsingleton $\omega$-limit sets. The $\omega$-limit sets of these essential points are, in a metric sense, stable under small perturbations of the function. We develop some of the properties of the essential point set of a continuous function, and investigate the relationship between essential points, $\omega$-limit sets, and the chaotic nature of the generating function.
\end{abstract}

\section{Introduction}

In Essential and non-essential fixed points, M. K. Fort, Jr. establishes the notion of an essential fixed point with the following definition: Let $x$ be a fixed point of $f$, a continuous self-map of $I=[0,1]$. If, for each neighborhood $U$ of $x$, there is an $\varepsilon>0$ so that $g: I \rightarrow I$ has a fixed point in $U$ whenever $\|f-g\|<\varepsilon$, then $x$ is an essential fixed point of $f$. Using the notion of semicontinuity and some of his earlier work involving set valued functions, Fort shows that the collection of essential functions found in $C(I, I)$ forms a residual set; that is, the typical element of $C(I, I)$ has the property that each of its fixed points is essential. Since the appearance of Fort's paper, several authors - to include Jiang, et al [12], S. Kinoshita [13], Y. Yonezawa [20], and Del Prete, et al [8] - have endeavored to extend or generalize his results. For the most part, these efforts have concentrated on extending Fort's results to spaces more general than $C(I, I)$, the collection of continuous self-maps of the unit interval. In this paper we work exclusively in $C(I, I)$ while extending Fort's concept of an essential point to points with nonsingleton $\omega$-limit sets.

We proceed through several sections. In section two we present the notation and definitions we will use throughout the balance of the paper. Paramount

Key Words: essential point, recurrent point, $\omega$-limit set

Mathematical Reviews subject classification: 54H20, 26A18

Received by the editors October 4, 1999 
among these is the following extension of Fort's notion of an essential point: Let $x$ be a recurrent point of $f$ in $C(I, I)$. We call $x$ an essential point of $f$ if for any $\varepsilon>0$ there exists a corresponding $\delta>0$ so that, whenever $g \in C(I, I)$ for which $\|f-g\|<\delta$, there exists $y$ a recurrent point of $g$ such that $\mathcal{H}(\omega(x, f), \omega(y, g))<\varepsilon$, where $\mathcal{H}$ denotes the Hausdorff metric. While perhaps not immediately apparent, we could just as well require that $y$ is a recurrent point of $g$ such that $|x-y|+\mathcal{H}(\omega(x, f), \omega(y, g))<\varepsilon$; that is, require $y$ to be close to $x$ as well as its $\omega$-limit set being close to $\omega(x, f)$. This is an equivalent formulation of an essential point, as we show in the proof of Proposition 17.

In section three we examine some of the elementary properties of a continuous function's set of essential points. We find that the essential point set of a function inherits many, but by no means all, of the properties of the recurrent point set, of which it is a subset. For example, while both sets are strongly invariant under the generating function, the essential point set of an iterate $f^{n}$ of $f$ may be a proper subset of the essential point set of $f$, whereas the recurrent sets of $f^{n}$ and $f$ are always equal. We also characterize essential periodic points of continuous functions using Bruckner's notion of stability [4]. This section concludes with a characterization of essential functions possessing zero topological entropy.

Section four is dedicated to the relationship between essential points and the $\omega$-limit sets to which they give rise. For example, we show that $\omega(x, f)$ must necessarily be a finite set, a Cantor set, or a union of finitely many nondegenerate closed intervals whenever $x$ is an essential point of $f$.

Our last section concerns the three maps defined on $C(I, I)$ that send a function to its collection of essential periodic points, recurrent points, and essential points. By considering the continuity structure of these maps, we are able to develop some insight into how perturbations affect a function's periodic, recurrent and essential point sets. We also find that essential functions are dense in the space of continuous self-maps of $I$ with zero topological entropy, a closed subset of $C(I, I)$.

\section{Preliminaries}

We shall be concerned with the class $C(I, I)$ of continuous functions mapping the unit interval $I=[0,1]$ into itself, and the properties associated with the essential point set of these functions. For $f$ in $C(I, I)$ and any integer $n \geq 1, f^{n}$ denotes the $n^{\text {th }}$ iterate of $f$. Let $P(f)$ represent those points $p \in I$ that are periodic under $f$, and if $p$ is a periodic point of period $n$ for which $f^{n}(p)-p$ is not unisigned in any deleted neighborhood of $p$, then $p$ is called a stable 
periodic point; we let $S(f)$ represent the stable periodic points of $f$. Those periodic points and stable periodic points of period $n$ are denoted by $P_{n}(f)$ and $S_{n}(f)$, respectively. Let $F(f)=\{x \in I: f(x)-x=0\}$ represent the fixed point set of $f$, so that $F_{n}(f)=F\left(f^{n}\right)=\left\{x \in I: f^{n}(x)-x=0\right\}=$ $\cup_{m \mid n} P_{m}(f)$. For each $x$ in $I$, we call the set of all subsequential limits of the trajectory $\left\{f^{n}(x)\right\}_{n=0}^{\infty}$ the $\omega$-limit set of $f$ generated by $x$, and write $\omega(x, f)$. Let $\Lambda(f)=\cup_{x \in I} \omega(x, f)$ represent the collection of $\omega$-limit points of $f$, while $\Omega(f)=\{\omega(x, f): x \in I\}$ denotes the set composed of the $\omega$-limit sets of $f$. If $x \in \omega(x, f)$, then we call $x$ a recurrent point of $f$, and write $x \in R(f)$; this is equivalent to saying that there exists a sequence $\left\{n_{k}\right\} \subset \mathbb{N}$ for which $\lim _{k \rightarrow \infty} f^{n_{k}}(x)=x$. Now, let $\varepsilon>0$ be given, and take $x$ and $y$ to be any points in $[0,1]$. An $\varepsilon$-chain from $x$ to $y$ with respect to a function $f$ is a finite set of points $\left\{x_{0}, x_{1}, \ldots, x_{n}\right\}$ in $[0,1]$ with $x=x_{0}, y=x_{n}$ and $\left|f\left(x_{k-1}\right)-x_{k}\right|<\varepsilon$ for $k=0,1, \ldots, n-1$. We call $x$ a chain recurrent point of $f$ if there is an $\varepsilon$-chain from $x$ to itself for any $\varepsilon>0$, and write $x \in C R(f)$.

In addition to the usual, Euclidean metric $d$ on $I=[0,1]$, we will be working in two metric spaces. Within $C(I, I)$ we will use the supremum metric given by $\|f-g\|=\sup \{|f(x)-g(x)|: x \in I\}$. Our second metric $\operatorname{space}(\mathcal{K}, \mathcal{H})$ is composed of the class of nonempty closed sets $\mathcal{K}$ in $I$ endowed with the Hausdorff metric $\mathcal{H}$ given by $\mathcal{H}(E, F)=\inf \left\{\delta>0: E \subset B_{\delta}(F), F \subset B_{\delta}(E)\right\}$, where $B_{\delta}(F)=\{x \in I: d(x, y)<\delta, y \in F\}$. This space is compact [5]. We are now in a position to generalize Fort's notion of an essential fixed point. For $f$ in $C(I, I)$, we call $x$ an essential point of $f$ if $x \in R(f)$, and for any $\varepsilon>0$ there exists a corresponding $\delta>0$ so that, whenever $g \in C(I, I)$ for which $\|f-g\|<\delta$, there exists $y$ a recurrent point of $g$ such that $\mathcal{H}(\omega(x, f), \omega(y, g))<$ $\varepsilon$. We call $f$ an essential function if $R(f)=E(f)$, the essential point set of $f$. The following result involving $\Omega(f)$ and $(\mathcal{K}, \mathcal{H})$ from [3] will be needed in the ensuing sections.

Theorem 1. For any $f$ in $C(I, I)$, the set $\Omega(f)$ is closed in $(\mathcal{K}, \mathcal{H})$.

In much of the sequel we will restrict our attention to a closed subset $\mathcal{E}$ of $C(I, I)$ composed of those functions $f$ having zero topological entropy, denoted by $\mathbf{h}(f)=0$. The reader is referred to Theorem $\mathrm{A}$ of [11] for an extensive list of equivalent formulations of topological entropy zero. For our purposes, it suffices to note that every periodic orbit of a continuous function with zero topological entropy has cardinality of a power of two. The following theorem, due to Smítal [15], sheds considerable light on the structure of infinite $\omega$-limit sets for functions with zero topological entropy.

Theorem 2. If $\omega$ is an infinite $\omega$-limit set of $f \in C(I, I)$ possessing zero topological entropy, then there exists a sequence of closed intervals $\left\{J_{k}\right\}_{k=1}^{\infty}$ in 
$[0,1]$ such that

1. for each $k,\left\{f^{i}\left(J_{k}\right)\right\}_{i=1}^{2^{k}}$ are pairwise disjoint, and $J_{k}=f^{2^{k}}\left(J_{k}\right)$.

2. for each $k, J_{k+1} \cup f^{2^{k}}\left(J_{k+1}\right) \subset J_{k}$.

3. for each $k, \omega \subset \cup_{i=1}^{2^{k}} f^{i}\left(J_{k}\right)$.

4. for each $k$ and $i, \omega \cap f^{i}\left(J_{k}\right) \neq \emptyset$.

We make the following definitions with Smítal's Theorem in mind. Let $\omega$ be an infinite compact subset of $I$, and let $f$ map $\omega$ into itself. We call $f$ a simple map on $\omega$ if $\omega$ has a decomposition $S \cup T$ into compact portions that $f$ exchanges, and $f^{2}$ is simple on each of these portions. From Smítal's Theorem one sees that every map $f$ with zero topological entropy is simple on each of its infinite $\omega$-limit sets. Let $\left\{J_{k}\right\}_{k=1}^{\infty}$ be a nested sequence of compact periodic intervals with respect to $\omega$ and $f$ as described in Smítal's Theorem. Every set of the form $\omega \cap f^{i}\left(J_{k}\right)$ is periodic of period $2^{k}$, and we call each such set a periodic portion of rank $k$. This system of periodic portions of $\omega$, or of the corresponding periodic intervals, is called the simple system of $\omega$ with respect to $f$. We now recall a device from [9] that allows us to code the sets $f^{i}\left(J_{k}\right)$ with finite tuples of zeros and ones. Let $\mathbb{N}$ denote the natural numbers, and take $\mathcal{N}$ to be the set of sequences composed of zeros and ones. If $\mathbf{n} \in \mathcal{N}$ and $\mathbf{n}=\left\{n_{i}\right\}_{i=1}^{\infty}$, we let $\mathbf{n} \mid k=\left(n_{1}, n_{2}, \ldots, n_{k}\right)$. Set $\mathbf{0}=\{0,0, \ldots\}$ and $\mathbf{1}=\{1,1, \ldots$.$\} . Now, define a function \mathcal{A}: \mathcal{N} \longrightarrow \mathcal{N}$ given by $\mathcal{A}(\mathbf{n})=\mathbf{n}+1 \mathbf{0}$, where addition is modulus two from left to right. For each $k \in \mathbb{N}$ and $i \in \mathbb{N}$ put $F_{\mathbf{1} \mid k}=J_{k}$ and $F_{\mathcal{A}^{i}(\mathbf{1} \mid k)}=f^{i}\left(J_{k}\right)$. Thus, for each $\mathbf{m}$ and $\mathbf{n}$ in $\mathcal{N}$ and $k \in \mathbb{N}$ there is a $j \in \mathbb{N}$ such that $\mathcal{A}^{j}(\mathbf{m} \mid k)=\mathbf{n} \mid k$; the above relations define $F_{\mathbf{n} \mid k}$ for all $\mathbf{n} \in \mathcal{N}$ and $k \in \mathbb{N}$. Now, set $F_{\mathbf{n}}=\cap_{k=1}^{\infty} F_{\mathbf{n} \mid k}$, and let $K=\cup_{\mathbf{n} \in \mathcal{N}} \cap_{k=1}^{\infty} F_{\mathbf{n} \mid k}$. Then $K$ and each $F_{\mathbf{n}}$ are compact, and the components of $K$ consist of the $F_{\mathbf{n}}$ sets.

Given the very specific behavior that functions of zero topological entropy must demonstrate on their infinite $\omega$-limit sets, it may not be too surprising that Bruckner and Smítal have been able to characterize these sets [7].

Theorem 3. An infinite compact set $W \subset(0,1)$ is an $\omega$-limit set of a map $f \in C(I, I)$ with zero topological entropy if and only if $W=Q \cup P$, where $Q$ is a Cantor set and $P$ is empty or countably infinite, disjoint with $Q$, and satisfies the following conditions:

- every interval contiguous to $Q$ contains at most two points of $P$;

- each of the intervals $[0, \min Q),(\max Q, 1]$ contains at most one point of $P$, and 
- $\bar{P}=Q \cup P$.

While the iterative structures of continuous functions with positive topological entropy can be quite involved, we will be able to make good use of one structure, called a horseshoe, that is shared by all these functions. If $f$ is a continuous self-map of $I$ for which $\mathbf{h}(f)>0$, then there exist compact subintervals $J$ and $K$ in $I$ with at most one common point, and a natural number $m$, for which $J \cup K \subset f^{m}(J) \cap f^{m}(K)$. Because of this there exists a compact set $X \subset I$ such that $f^{m}(X)=X$, and $f^{m} \mid X$ is semiconjugate to the shift operator $\sigma$ on two symbols [2].

Positive topological entropy provides one notion of chaos; another concept of chaos comes from Li and Yorke [14]. We say a function $f \in C(I, I)$ is chaotic in the sense of $\mathrm{Li}$ and Yorke if there exists an uncountable set $X \subset I$ so that for any pair of distinct points $x$ and $y$ in $X, \liminf _{n \rightarrow \infty}\left|f^{n}(x)-f^{n}(y)\right|=0$ and $\lim \sup _{n \rightarrow \infty}\left|f^{n}(x)-f^{n}(y)\right|>0$. The existence of a horseshoe $X \subset I$ insures that every function $f$ for which $\mathbf{h}(f)>0$ is chaotic in the sense of $\mathrm{Li}$ and Yorke. As Bruckner and Ceder show [6], a function $f$ with zero topological entropy is chaotic in the sense of $\mathrm{Li}$ and Yorke if and only if int $K \neq \emptyset$ for some simple system of $f$.

\section{The Set of Essential Points}

We begin with a rather straightforward geometric characterization of essential periodic points. This result uses Bruckner's notion of stability [4], and generalizes the characterization of an essential fixed point found in [8] in a natural way.

Proposition 4. If $f \in C(I, I)$, then $z$ is an essential periodic point of $f$ if and only if $z \in S(f)$.

Proof. Suppose $z \in S_{n}(f)$ so that $f^{n}(z)-z$ is not unisigned in any deleted neighborhood of $z$. Let $\varepsilon>0$. Thus, for every $g \in C(I, I)$ sufficiently close to $f$, there exist $x, y$ in $B_{\frac{\varepsilon}{2}}(z)$ so that $g^{n}(x)>x$ and $g^{n}(y)<y$, and $P_{n}(g) \cap$ $B_{\frac{\varepsilon}{2}}(z) \neq \emptyset$. By taking $\delta<\frac{\varepsilon}{2}$ if necessary, we may insure the existence of $x \in P_{n}(g) \cap B_{\delta}(z)$ whenever $\|f-g\|<\delta$ so that $\mathcal{H}(\omega(x, g), \omega(y, g))<\varepsilon$. It follows that $z$ is an essential point of $f$.

Now, let us suppose $z \in P_{n}(f)$ is an interior point of $I$, and there exists $U$ a neighborhood of $z$ so that $f^{n}(x)-x \geq 0$ for any $x \in U$. The proof concerning the endpoints is similar. By taking a smaller neighborhood of $z$ if necessary, we may presume $f^{n}(x) \neq 1$ for any $x \in U$, too. Now, let $V$ be a neighborhood of $z$ contained in $U$ so that $V, f(V), f^{2}(V), \ldots, f^{n-1}(V)$ are disjoint, and $f^{n}(V) \subset$ 
$U$. Let $\varepsilon>0$, and set $a=\inf \left\{f^{n-1}(x): x \in V\right\}, b=\sup \left\{f^{n-1}(x): x \in V\right\}$. Let $g \in C(I, I)$ for which $\|f-g\|<\varepsilon, g(x)>f(x)$ on $[a, b], g^{n}(\bar{V}) \subset U$ and $V, g(V), g^{2}(V), \ldots, g^{n-1}(V)$ are disjoint. Then $F\left(g^{n}\right) \cap V=\emptyset$, and $z$ is not essential.

We now turn our attention to results concerning the structure and properties of the set of recurrent points and the set of essential points of a continuous self-map of the interval. We see that, while $E(f)$ inherits many of $R(f)$ 's nice properties, this is by no means always the case. We begin with a result that relates the structure of the recurrent point set of a function to its topological entropy. While this result must be known, the author is unable to provide a reference.

Lemma 5. If $f \in C(I, I)$, then $R(f)$ is closed if and only if $\mathbf{h}(f)=0$.

Proof. Suppose $\mathbf{h}(f)=0$. From Proposition IV.15 of [2], we know that $R(f) \subset \overline{P(f)}$ for any $f \in C(I, I)$. Let $\left\{x_{n}\right\} \subset R(f)$ so that $x_{n} \rightarrow x$. Then there exists $\left\{y_{n}\right\} \subset P(f)$ such that $y_{n} \rightarrow x$, and since $\Omega(f)$ is closed in $(\mathcal{K}, \mathcal{H})$ compact, we may also assume that $\omega\left(y_{n}, f\right) \rightarrow \omega \in \Omega(f)$, with $x \in \omega$. If $x$ is not contained in $R(f)$, then $x$ cannot be a periodic point, since $P(f) \subset R(f)$ by definition. If $\omega$ is a Cantor set, then $\omega(y, f)=\omega$ for all $y \in \omega$ by Proposition $3.1(7)$ of [6]. If $x$ is not a recurrent point, then, it follows that $\omega$ must be infinite, and by Theorem 3 that $\omega=Q \cup P$, where $Q$ is a Cantor set and $P$ is nonempty and countably infinite. Moreover, $x$ is an element of $P$ : if $x \in Q$, then $\omega(x, f)=Q$, a proper subset of $Q \cup P$, and $x \in R(f)$, again by Proposition 3.1(7) [6]. But $x \in P$ contradicts the existence of $\left\{y_{n}\right\} \subset P(f)$ for which $y_{n} \rightarrow x$, as parts (6) and (13) of Proposition 3.1 [6] show that $P \cap \overline{P(f)}=\emptyset$.

Now, suppose $\mathbf{h}(f)>0$. Then there exists $X \subset I$ and $m$ a natural number so that $f^{m}: X \rightarrow X$ is semiconjugate to the shift operator $\sigma$ on two symbols, so that $X$ is not contained in $R(f)$. Since $P(f) \subset R(f)$ and $X \subset \overline{P(f)}, R(f)$ is not closed.

In a similar fashion one sees that the set of essential points of a function is also closed if and only if the function has zero topological entropy. In the course of our proof we presume that $S\left(f^{m}\right) \subset S(f)$; we prove this in Proposition 13 .

Theorem 6. If $f \in C(I, I)$, then $E(f)$ is closed if and only if $\mathbf{h}(f)=0$.

Proof. Suppose $\mathbf{h}(f)=0,\left\{x_{n}\right\} \subset E(f)$ and $x_{n} \rightarrow x$. Since $\Omega(f)$ is closed in the compact metric space $(\mathcal{K}, \mathcal{H})$, we may presume that $\omega\left(x_{n}, f\right) \rightarrow \omega \in \Omega(f)$, where $x \in \omega$. Suppose now that $x$ is not an element of $R(f)$. Then $\omega=P \cup Q$, where $P$ is a Cantor set and $Q$ is countable, with $x \in Q$. If $\omega\left(x_{n}, f\right)$ is infinite, then $\omega\left(x_{n}, f\right)$ is perfect, so there is $y_{n} \in P(f)$ so that $\mathcal{H}\left(\omega\left(x_{n}, f\right), \omega\left(y_{n}, f\right)\right)<$ 
$\frac{1}{n}$; thus there exists $\left\{y_{n}\right\} \subset P(f)$ such that $y_{n} \rightarrow x$. But this contradicts $x$ being an element of $Q$, so that $x \in R(f)$. Moreover, since $\mathbf{h}(f)=0$, it follows that $\omega=\omega(x, f)$. It remains to show that $x$ is essential. Let $\varepsilon>0$. There exists $N$ a natural number so that $\mathcal{H}\left(\omega\left(x_{N}, f\right), \omega(x, f)\right)<\frac{\varepsilon}{2}$, and there exists $\delta>0$ so that $\|f-g\|<\delta$ implies the existence of $y \in R(g)$ so that $\mathcal{H}\left(\omega\left(x_{N}, f\right), \omega(y, g)\right)<\frac{\varepsilon}{2}$. Thus, $\mathcal{H}(\omega(x, f), \omega(y, g))<\varepsilon$, and our conclusion follows.

Suppose $\mathbf{h}(f)>0$, and let $X \subset I$ so that $f^{m} \mid X$ is semiconjugate to the shift operator $\sigma$ on two symbols, for some natural number $m$. Since $X$ contains a homoclinic trajectory, $X$ is not contained in $R\left(f^{m}\right)=R(f)$ [2]. Since $X \subset \overline{S\left(f^{m}\right)}$, and $S\left(f^{m}\right) \subset S(f)$, it follows that $X \subset \overline{S(f)}$. Thus $\overline{S(f)}$ is not a subset of $E(f)$, so that $E(f)$ is not closed.

Before considering a couple interesting consequences of our lemma, we develop an example to show just how badly the set of essential points can fail to be closed when one considers a function with positive topological entropy.

Example 7. There exists $f \in C(I, I)$, necessarily possessing positive topological entropy, and a sequence of essential periodic orbits $\left\{\omega\left(x_{k}, f\right)\right\}_{k=1}^{\infty}$ in $\Omega(f)$ for which $\omega=\lim _{k \rightarrow \infty} \omega\left(x_{k}, f\right)$ exists, yet no point of $\omega$ is essential.

Construction. Let $h:[0,1] \rightarrow[0,1]$ be the hat function given by $x \longmapsto 2 x$ if $x \in\left[0, \frac{1}{2}\right]$ and $x \longmapsto 2(1-x)$ if $x \in\left[\frac{1}{2}, 1\right]$. Then $h^{2^{k}}$ has a zero at $\frac{l}{2^{2^{k}}}$ if $2 \mid l$ and $h^{2^{k}}$ is one at $\frac{l}{2^{2^{k}}}$ if 2 does not divide $l$, for $l=0,1, \ldots, 2^{2^{k}}$. To get the entire graph of $h^{2^{k}}$ we extend it linearly on each of the intervals $\left[\frac{m}{2^{2^{k}}}, \frac{m+1}{2^{2^{k}}}\right]$, for $m=0,1, \ldots, 2^{2^{k}}-1$. It follows that $h$ has an essential periodic point of period $2^{k}$ on the interval $\left[\frac{1}{2^{2^{k}}}, \frac{1}{2^{2^{k}-1}}\right]$; call this point $x_{k}$. Since $(\mathcal{K}, \mathcal{H})$ is a compact metric space, there exists $\left\{x_{k_{j}}\right\} \subset\left\{x_{k}\right\}$ so that $\lim _{j \rightarrow \infty} \omega\left(x_{k_{j}}, h\right)$ exists; call this limit set $\omega$. Since $\Omega(h)$ is closed, $\omega \in \Omega(h)$, and because $x_{k_{j}} \rightarrow 0$, we know that $0 \in \omega$. Since $\{0\}$ is not an essential point of $h$, it follows that the set of essential points of $h$ is not closed. In fact, a tedious but not too difficult calculation shows that we can take $\left\{x_{k_{j}}\right\}=\left\{x_{k}\right\}$ and $\omega=\{0\} \cup\left\{\cup_{k=0}^{\infty} \frac{1}{2^{k}}\right\}$, so that $\omega$ is a homoclinic trajectory of $h$. Since $\omega(x, h)=\{0\}$ for any $x \in \omega$, one sees that $\{0\}=R(h) \cap \omega$. Because $\{0\}$ is not contained in $E(f), \omega \cap E(f)=\emptyset$. It is worth noting that 0 is the unique periodic point of $h$ that is not stable.

We now consider a couple corollaries to Proposition 6.

Corollary 8. If $f \in C(I, I)$ and $\mathbf{h}(f)=0$, then $\overline{S(f)} \subset E(f)$.

It may, in fact, be true that $\overline{S(f)}=E(f)$ whenever $f$ is a continuous function with zero topological entropy, but the author is unable to either prove this or develop an appropriate counterexample. 
Corollary 9. If $f \in C(I, I), \mathbf{h}(f)=0$ and $x \in R(f)$ for which $\omega(x, f)$ is infinite, then $x$ is an essential point.

Proof. Since $x \in R(f)$, it follows that $\omega(x, f)$ is a nowhere dense perfect set, so that there exists $\omega_{n} \in \Omega(f)$ such that $\left|\omega_{n}\right|=2^{n}$ for any $n$, and $\mathcal{H}\left(\omega_{n}, \omega(x, f)\right) \rightarrow 0$ as $n \rightarrow \infty$. Let $\varepsilon>0$, and choose $N$ a natural number so that $\mathcal{H}\left(\omega_{N}, \omega(x, f)\right)<\frac{\varepsilon}{2}$. Moreover, we may choose our $N$ so that there is a $\delta>0$ for which $\|f-g\|<\delta$ implies the existence of $y$ a periodic point of $g$ with $\mathcal{H}\left(\omega_{N}, \omega(y, g)\right)<\frac{\varepsilon}{2}$, by Theorem II.19 of [2]. It follows that $y \in R(g)$ for which $\mathcal{H}(\omega(y, g), \omega(x, f))<\varepsilon$.

Our next result shows that, just as the set of recurrent points of a continuous function is strongly invariant, so is the set of essential points.

Proposition 10. If $f \in C(I, I)$, then $f(E(f))=E(f)$.

Proof. Suppose $x \in E(f)$, and $y=f(x)$. Then $y \in \omega(x, f)=\omega(f(x), f)=$ $\omega(y, f)$, so that $y \in R(f)$. Let $\varepsilon>0$. Since $x \in E(f)$, there exists a $\delta>0$ so that $\|f-g\|<\delta$ implies the existence of $z$ a recurrent point of $g$ for which $\mathcal{H}(\omega(z, g), \omega(x, f))=\mathcal{H}(\omega(z, g), \omega(y, f))<\varepsilon$. Thus, $y \in E(f)$, and $f(E(f)) \subset E(f)$.

Now, suppose $x \in E(f)$. Then $x \in \omega(x, f)$, and since $\omega(x, f)$ is strongly invariant under $f$, there is a $y$ in $\omega(x, f)$ for which $f(y)=x$. Since $y$ must be an essential point of $f$, it follows that $f(E(f)) \supset E(f)$.

As mentioned earlier, $E(f)$ inherits some, but not all, of the nice properties $R(f)$ possesses. We turn our attention to some of these differences. We first show that, while $R\left(f^{n}\right)=R(f)$ for any natural number $n$, the inclusion can be proper when one considers essential points; this is true even for functions with zero topological entropy.

Proposition 11. For any $f$ in $C(I, I)$ and for any natural number $n, E\left(f^{n}\right) \subset$ $E(f)$.

Proof. Since $x \in E\left(f^{n}\right)$, it follows that $x \in \omega\left(x, f^{n}\right)$. Since $\omega\left(x, f^{n}\right) \subset$ $\omega(x, f)$ for any $n \in \mathbb{N}$, we have that $x \in \omega(x, f)$, so that $x \in E(f)$.

Example 12. There exists a function $f$ possessing zero topological entropy for which $E\left(f^{n}\right)$ is a proper subset of $E(f)$ for infinitely many natural numbers $n$.

Construction. Let $f \in C(I, I)$ be given by $f(x)=1-x$. Then $y=\frac{1}{2}$ is an essential fixed point of $f$, and since $f^{2}(x)=1-(1-x)=x$, we see that $f^{2 k}$ is the identity function for all $k$ in $\mathbb{N}$. 
In a similar fashion, $S\left(f^{n}\right)$ can be a proper subset of $S(f)$, even though $P(f)$ and $P\left(f^{n}\right)$ are always equal. Our next proposition confirms that $S\left(f^{m}\right) \subset$ $S(f)$ for all natural numbers $m$, a fact assumed without proof in the verification of Theorem 6 . One can also view this proposition as a corollary to Proposition 11.

Proposition 13. Let $f \in C(I, I)$. If $y \in P_{n}(f)$, and there exists $m=k n a$ natural number so that $f^{m}(x)-x$ is not unisigned in any deleted neighborhood of $y$, then $y$ is an essential periodic point.

Proof. Suppose, to the contrary, that $y$ is not an essential periodic point of $f$, so that there exists $U$ a neighborhood of $y$ on which $f^{n}(x)-x$ is unisigned. Say $f^{n}(x)-x \geq 0$ for any $x$ in $U$. Since $f^{n}$ is uniformly continuous, there exists $V \subset U$ a neighborhood of $x$ so that $f^{j n}(V) \subset U$, for $j=1,2, \ldots, k$. That $f^{k n}(x) \geq x$ for any $x \in V \subset U$ follows from the fact that $f^{n}(x)-x \geq 0$ for any $x$ in $U$. But this implies $f^{m}(x)-x$ is unisigned on $V$.

We close this section with a characterization of essential functions with zero topological entropy.

Theorem 14. If $f \in C(I, I)$ and $\mathbf{h}(f)=0$, then $f$ is an essential function if and only if every periodic point of $f$ is essential.

Proof. Suppose every periodic point of $f$ is essential. Since $R(f) \subset \overline{P(f)}$, it suffices to show that $x \in R(f)-P(f)$ is an essential point. But this implies that $\omega(x, f)$ is infinite, so our conclusion follows from Corollary 9.

If $f$ is an essential map, then every point of $R(f)$ must be essential. But $P(f) \subset R(f)$, so every periodic point of $f$ is essential.

\section{Essential Points and $\omega$-Limit Sets}

We now turn our attention to a brief discussion of essential points, and their relationship to the function's $\omega$-limit sets. Our first result deals with the types of $\omega$-limit sets that can be generated by the recurrent points of a continuous function. We get an analogous result concerning essential points as a corollary.

Proposition 15. If $f \in C(I, I)$ and $x$ is a recurrent point of $f$, then $\omega(x, f)$ must be either finite or perfect. That is, $\omega(x, f)$ must be either a periodic orbit, a Cantor set, or a union of finitely many nondegenerate closed intervals.

Proof. Let $y \in \omega(x, f)$, an infinite $\omega$-limit set of $f$. It suffices to show that $y$ is not isolated in $\omega(x, f)$ for our conclusion to follow. Since $x \in \omega(x, f)$ and $\omega(x, f)$ is strongly invariant, ${\overline{\left\{f^{n}(x)\right\}_{n=0}^{\infty}}}_{n}^{\infty} \omega(x, f)$. It follows, then, that a 
subsequence of $\left\{f^{n}(x)\right\}_{n=0}^{\infty}$ converges to $y$, so that $y$ is not isolated in $\omega(x, f)$. The structures possible for $\omega(x, f)$ now follow from [1].

Since by definition $E(f)$ is a subset of $R(f)$, we have the following corollary concerning essential points, and the structure of their $\omega$-limit sets.

Corollary 16. If $f \in C(I, I)$ and $x$ is an essential point of $f$, then $\omega(x, f)$ must be either finite, a Cantor set, or a union of finitely many nondegenerate closed intervals.

The following results deal with essential points and those $\omega$-limit sets generated by an essential point. We find that while all of $\omega(x, f)$ need not be contained in $E(f)$ whenever $x \in E(f)$, there is always a subset dense in the $\omega$-limit set that is composed of essential points. Our first proposition along these lines is rather technical; its value stems from the corollaries that follow. We also take this opportunity to show that $x \in E(f)$ if and only if, for any $\varepsilon>0$, there is a corresponding $\delta>0$ so that $\|f-g\|<\delta$ implies the existence of $y \in R(g)$ so that $|x-y|<\varepsilon$ as well as $\mathcal{H}(\omega(x, f), \omega(y, g))<\varepsilon$. That is, we can require $y$ to be a recurrent point of $g$ that not only has an $\omega$-limit set that is $\varepsilon$-close to $\omega(x, f)$, but that $y$ itself is $\varepsilon$-close to $x$.

Proposition 17. Let $f \in C(I, I)$, with $x \in E(f)$. If $y \in \omega(x, f)$ for which $\omega(x, f)=\omega(y, f)$, then $y \in E(f)$.

Proof. Since $y \in \omega(y, f)=\omega(x, f)$, it follows that $y \in R(f)$. Since $x \in E(f)$, for any $\varepsilon>0$ there exists a $\delta>0$ so that $\|f-g\|<\delta$ implies the existence of $z$ a recurrent point of $g$ so that $\mathcal{H}(\omega(z, g), \omega(x, f))<\frac{\varepsilon}{2}$. Thus, there is an element $a$ of $\omega(z, g)$ so that $|y-a|<\frac{\varepsilon}{2}$. Since $z \in R(g)$ and $\omega(z, g)$ is strongly invariant under $g$, we have that $g^{n}(z) \in \omega\left(g^{n}(z), g\right)=\omega(z, g)$ for any $n$, so

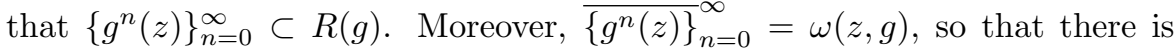
$b \in R(g)$ such that $|a-b|<\frac{\varepsilon}{2}$, and $\omega(b, g)=\omega(z, g)$. Thus, $|y-b|<\varepsilon$, and $y \in E(f)$.

As a consequence of Proposition 17, we have the following two corollaries, the first of which follows from Proposition 10, too.

Corollary 18. If $f \in C(I, I)$ and $x \in E(f)$, then $\left\{f^{n}(x)\right\}_{n=0}^{\infty} \subset E(f)$.

Corollary 19. If $f \in C(I, I), \mathbf{h}(f)=0$ and $x \in E(f)$, then $\omega(x, f) \subset E(f)$.

Proof. Suppose $x \in E(f)$, and $\omega(x, f)$ is infinite. Then $\omega(x, f)$ is a Cantor set, and by Smítal's Theorem, $\omega(x, f)=\omega(y, f)$ for every $y \in \omega(x, f)$.

A consideration of Example 7 shows that one cannot extend Corollary 19 to functions with positive topological entropy. Suppose $\mathbf{h}(f)>0$ with $X \subset I$ 
and $m \in \mathbb{N}$ so that $f^{m} \mid X$ is semiconjugate to the shift operator $\sigma$ on two symbols. Since there exists a sequence of stable periodic orbits $\left\{\omega_{n}\right\} \subset \Omega\left(f^{m}\right)$ so that $\omega_{n} \rightarrow X$, there exists $x \in E\left(f^{m}\right)$ for which $\omega\left(x, f^{m}\right)=X$. But $X$ is not a subset of $E(f)$.

\section{Set Valued Functions}

Some of the more interesting results found in Fort's paper relate the structure of the fixed point set of a continuous function $f$ to the behavior of the map $F:(C(I, I),\|\circ\|) \rightarrow(\mathcal{K}, \mathcal{H})$ given by $g \longmapsto F(g)$ at the function $f$. Since we will concern ourselves with generalizations of these results, for the sake of clarity and convenience, we restate Fort's results below. The first of these can also be found in [19].

Theorem 20. The function $F:(C(I, I),\|\circ\|) \rightarrow(\mathcal{K}, \mathcal{H})$ given by $g \longmapsto F(g)$ is upper semicontinuous.

Theorem 21. If $f \in C(I, I)$, then each fixed point of $f$ is essential if and only if $f$ is a point of continuity of the map $F:(C(I, I),\|\circ\|) \rightarrow(\mathcal{K}, \mathcal{H})$.

Theorem 22. If $f \in C(I, I)$ and $\varepsilon>0$, then there exists $g$ in $C(I, I)$ so that $\|f-g\|<\varepsilon$, and each fixed point of $g$ is essential.

We begin by considering the map $F_{n}:(C(I, I),\|\circ\|) \rightarrow(\mathcal{K}, \mathcal{H})$ given by $g \longmapsto F_{n}(g)=F\left(g^{n}\right)$. By making relatively straightforward changes to the proofs found in [10], one gets the following series of corollaries.

Corollary 23. The function $F_{n}:(C(I, I),\|\circ\|) \rightarrow(\mathcal{K}, \mathcal{H})$ is upper semicontinuous.

Corollary 24. If $f \in C(I, I)$, then each point of $F_{n}(f)$ is essential if and only if $f$ is a point of continuity of the map $F_{n}:(C(I, I),\|\circ\|) \rightarrow(\mathcal{K}, \mathcal{H})$.

Corollary 25. If $f \in C(I, I)$ and $\varepsilon>0$, then there exists $g$ in $C(I, I)$ so that $\|f-g\|<\varepsilon$, and each point of $F_{n}(g)$ is essential.

The following result also follows from Theorem 21, and characterizes essential $2^{n}$-functions.

Corollary 26. If $f \in C(I, I)$ is a $2^{n}$-function, then $f$ is essential if and only if the map $F_{2^{n}}:(C(I, I),\|\circ\|) \rightarrow(\mathcal{K}, \mathcal{H})$ is continuous at $f$.

Let us now consider the map $R:(\mathcal{E},\|\circ\|) \rightarrow(\mathcal{K}, \mathcal{H})$ given by $g \longmapsto R(g)$. In a sense, this map is more general than $F_{n}:(C(I, I),\|\circ\|) \rightarrow(\mathcal{K}, \mathcal{H})$ since 
$F_{n}(g) \subset R(g)$ for all natural numbers $n$. We have, however, severely restricted the domain of $R:(\mathcal{E},\|\circ\|) \rightarrow(\mathcal{K}, \mathcal{H})$ by considering only those functions with zero topological entropy. With Fort's Theorem 20 in mind, we begin with the following.

Proposition 27. The function $R:(\mathcal{E},\|\circ\|) \rightarrow(\mathcal{K}, \mathcal{H})$ given by $g \longmapsto R(g)$ is upper semicontinuous at $f$ if and only if $f$ is nonchaotic in the sense of $L i$ and Yorke.

Proof. If $f$ is not chaotic in the sense of Li and Yorke, then $C R(f)=\Lambda(f)=$ $R(f)$ [16]. Now, suppose that there exists $\left\{f_{n}\right\} \subset \mathcal{E}$ so that $f_{n} \rightarrow f$ with $x_{n} \in R\left(f_{n}\right)$ for any $n$, and $x_{n} \rightarrow x$. Since we may presume that $x_{n} \in P\left(f_{n}\right)$ for each $n$, we see that $x \in C R(f)=R(f)$.

Now, suppose $f \in \mathcal{E}$ is chaotic in the sense of Li and Yorke. Then int $K \neq \emptyset$ for some simple system of $f[6]$, so that $\Lambda(f)$ is a proper subset of $C R(f)$. It follows that $R:(\mathcal{E},\|\circ\|) \rightarrow(\mathcal{K}, \mathcal{H})$ is not upper semicontinuous at $f$ [16].

Our next two results generalize, in some sense, Theorem 21. They show that while continuity of $R:(\mathcal{E},\|\circ\|) \rightarrow(\mathcal{K}, \mathcal{H})$ at $f$ is sufficient to insure that $f$ is an essential function, this condition is not necessary.

Proposition 28. If $R:(\mathcal{E},\|\circ\|) \rightarrow(\mathcal{K}, \mathcal{H})$ is continuous at $f$, then $f$ is an essential function.

Proof. Let $x \in R(f)$. We show that $x$ is essential. Since $R:(\mathcal{E},\|\circ\|) \rightarrow$ $(\mathcal{K}, \mathcal{H})$ is continuous at $f$, for any $\left\{f_{n}\right\} \subset \mathcal{E}$ such that $f_{n} \rightarrow f$, there exists $x_{n} \in R\left(f_{n}\right)$ so that $x_{n} \rightarrow x$. Moreover, as $(\mathcal{K}, \mathcal{H})$ is compact, we may assume that $\left\{\omega\left(x_{n}, f_{n}\right)\right\}$ converges; say $\omega=\lim _{n \rightarrow \infty} \omega\left(x_{n}, f_{n}\right)$. Then $x \in \omega$. Now, let us suppose that $\omega$ is finite. By Theorem 6 of [17], it follows that $\omega$ is a periodic orbit of $f$, so that $\omega=\omega(x, f)$, and our desired conclusion follows. Suppose, on the other hand, that $\omega$ is infinite. Since $f(\omega)=\omega$ by Lemma 4 of [17], we know that $\omega(x, f) \subset \omega$, and if $C$ is the set of isolated points of $\omega$, them $\omega-C$ is an $\omega$-limit set of $f$. Because $\mathbf{h}(f)=0$ and $x \in R(f)$, it follows that $x \in \omega-C$ and that $\omega(x, f)=\omega-C$. Since $\omega(x, f)$ is infinite and $\mathbf{h}(f)=0$, it follows that $x$ is essential.

The following lemma shows that $R:(\mathcal{E},\|\circ\|) \rightarrow(\mathcal{K}, \mathcal{H})$ need not be continuous at a function in order for that function to be essential.

Lemma 29. There exist functions in $\mathcal{E}$ that are essential, but at which the map $R:(\mathcal{E},\|\circ\|) \rightarrow(\mathcal{K}, \mathcal{H})$ is not continuous.

Proof. Let $f: I \rightarrow I$ be a continuous function for which $\mathbf{h}(f)=0$ and $S(f)=P(f)$, but $f$ is chaotic in the sense of $\mathrm{Li}$ and Yorke. This is equivalent 
to requiring that there exists a simple system of $f$ for which int $K \neq \emptyset$, or that $R(f)$ is a proper subset of $C R(f)$.

Turning our attention to the map $E:(\mathcal{E},\|\circ\|) \rightarrow(\mathcal{K}, \mathcal{H})$, we again show that continuity at a function is a sufficient, but not a necessary condition, in order for that function to be essential.

Proposition 30. If $E:(\mathcal{E},\|\circ\|) \rightarrow(\mathcal{K}, \mathcal{H})$ given by $g \longmapsto E(g)$ is continuous at $f$, then $f$ is an essential function.

Proof. Suppose $E:(\mathcal{E},\|\circ\| \rightarrow(\mathcal{K}, \mathcal{H})$ is continuous at $f$. We wish to show that $R(f)=E(f)$. If $x \in R(f)$ and $\omega(x, f)$ is infinite, then $x$ is essential. Let us presume, then, that $x \in R(f)$ and $\omega(x, f)$ is a periodic $\omega$-limit set. Suppose $f_{n} \rightarrow f$. Then there exists a sequence of points $\left\{x_{n}\right\}$ such that $x_{n} \in E\left(f_{n}\right) \subset$ $R\left(f_{n}\right)$ for each $n, \lim _{n \rightarrow \infty} x_{n}=x$ and $\lim _{n \rightarrow \infty} \omega\left(x_{n}, f_{n}\right)=\omega(x, f)$. It follows that $x$ is essential.

Lemma 31. There exist functions in $\mathcal{E}$ that are essential, but at which the map $E:(\mathcal{E},\|\circ\|) \rightarrow(\mathcal{K}, \mathcal{H})$ is not continuous.

Proof. Let $f: I \rightarrow I$ be a continuous function for which $\mathbf{h}(f)=0$ and $S(f)=P(f)$, but $f$ is chaotic in the sense of Li and Yorke. Then there exists a simple system of $f$ for which int $K \neq \emptyset$, or that $R(f)$ is a proper subset of $C R(f)$. Now, apply the example found in Lemma 16 of [16]. This allows us to find, for any $\varepsilon>0$, a function $g$ in $\mathcal{E}$ so that $S(g) \cap$ int $K \neq \emptyset$, and $\|f-g\|<\varepsilon$. Since int $K \subset C R(f)-R(f)$, this shows that $E$ is not upper semicontinuous at $f$, so that $E$ cannot be continuous there.

We conclude with a generalization of Fort's Theorem 22 to a function's set of essential points. Since the map $E:(\mathcal{E},\|\circ\|) \rightarrow(\mathcal{K}, \mathcal{H})$ is not upper semicontinuous, our proof must rest on ideas quite different than those of Fort. Nevertheless, we conclude that essential functions comprise a dense subset of $\mathcal{E}$.

Theorem 32. If $f$ is an element of $\mathcal{E}$ and $\varepsilon>0$, then there exists $g$ in $\mathcal{E}$ so that $\|f-g\|<\varepsilon$, and $g$ is an essential function.

Proof. Let $\varepsilon>0$. From Theorem 4 of [18], there exists $n$ a natural number and $h$ a $2^{n}$-function such that $\|f-h\|<\frac{\varepsilon}{2}$. Using Corollary 25, let $g$ be an essential $2^{n}$-function such that $\|g-h\|<\frac{\varepsilon}{2}$. Then $\|f-g\|<\varepsilon$, and our conclusion follows. 


\section{References}

[1] S. Agronsky, A. Bruckner, J. Ceder and T. Pearson, The structure of $\omega$-limit sets for continuous functions, Real Anal. Ex. 15 (1989), 483-510.

[2] L. Block and W. Coppel, Dynamics in one dimension, Lecture Notes in Mathematics, vol. 1513, Springer-Verlag, 1991.

[3] A. Blokh, A. M. Bruckner, P. D. Humke and J. Smítal, The space of $\omega$ limit sets of a continuous map of the interval, Trans. Amer. Math. Soc. 348 (1996), 1357-1372.

[4] A.M. Bruckner, Stability in the family of $\omega$-limit sets of continuous self maps of the interval, Real Anal. Ex. 22 (1997), 52-57.

[5] A. M. Bruckner, J. B. Bruckner and B. S. Thomson, Real analysis, Prentice-Hall, 1997.

[6] A. M. Bruckner and J. G. Ceder, Chaos in terms of the map $x \longmapsto \omega(x, f)$, Pac. J. Math. 156 (1992), 63-96.

[7] A. M. Bruckner and J. Smítal, The structure of $\omega$-limit sets of maps of the interval with zero topological entropy, Ergod. Th. and Dynam. Sys. 13 (1993), 7-19.

[8] I. Del Prete, M. Di Iorio and S. Naimpally, Essential fixed points of functions and multifunctions, preprint, 1998.

[9] R. L. Devaney, Chaotic dynamical systems, Benjamin/Cummings Publ. Co., 1986.

[10] M. K. Fort, Jr., Essential and nonessential fixed points, Amer. J. Math. 72 (1950), 315-322.

[11] V. Fedorenko, A. Sarkovskii and J. Smítal, Characterizations of weakly chaotic maps of the interval, Proc. Amer. Math. Soc. 110 (1990), 141148.

[12] Jiang and Jia-he, Essential component of the set of fixed points of multivalued mappings and its application to the theory of games, Sci. Sinica 12 (1963), 951-964.

[13] S. Kinoshita, On essential components of the set of fixed points, Osaka Math. J. 4 (1952), 19-22. 
[14] T. Li and J. Yorke, Period three implies chaos, Amer. Math. Monthly 82 (1975), 985-992.

[15] J. Smítal, Chaotic functions with zero topological entropy, Trans. Amer. Math. Soc. 297 (1986), 269-282.

[16] T. H. Steele, Continuity structure of $f \longmapsto \cup_{x \in I} \omega(x, f)$ and $f \longmapsto$ $\{\omega(x, f): x \in I\}$, Real Anal. Ex. 25 (2000), 421-429.

[17] T. H. Steele, Iterative stability in the class of continuous functions, Real Anal. Ex. 24 (1999), 765-780.

[18] T. H. Steele, The set of continuous functions with zero topological entropy, Real Anal. Ex. 24 (1999), 821-826.

[19] J. V. Wehausen, Transformations in metric spaces and ordinary differential equations, Bull. Amer. Math. Soc. 51 (1945), 113-119.

[20] Y. Yonezawa, On f.p.p. and $f^{*}$.p.p. of some not locally connected continua, Fund. Math. 139 (1991), 91-98. 
T. H. Steele 